\title{
Turkish cases of Mabry Syndrome with a novel homozygous mutation in PGAP2 gene
}

\begin{abstract}
Hyperphosphatasia with mental retardation syndrome is a genetic disorder. We report two siblings aged three years and fourteen years who were investigated for global development delays, seizures and dysmorphic features. A novel missense variant, c.1003G >A (p. Ala335 Thr chr11.3,846,572 NM_001256236.1), in PGAP2 gene was identified using whole-exome sequencing. We highlight the significance of elevated alkaline phosphatase in patients with certain dysmorphic features, can lead to the diagnosis of hyperphosphatasia with mental retardation syndrome using exome sequencing.
\end{abstract}

\section{Keywords}

Hyperphosphatasia with mental retardation syndrome, PGAP2, exome sequencing, spastic quadriplegia, alkaline phosphatase

\section{Introduction}

Hyperphosphatasia with mental retardation syndrome (HPMRS) also known as Mabry syndrome, is an autosomal recessive disorder characterised by intellectual disability and elevated levels of serum alkaline phosphatase (ALP). It is accompanied by seizures, facial dysmorphism and various anomalies. So far, six genes including PIGV, PIGO, PGAP2, PGAP3, PIGW, and PIGY involved in GPI(Glycosylphosphatidylinositol) biosynthesis, have been reported in association with HPMRS [1][2]. PGAP2 is one of the most recent identified genes in association with HPMRS. We report two siblings with novel missense variant and present new clinical findings which further describes characteristics of this rare disorder in humans.

\section{Case 1}

The patient was a 3-year-old boy who was referred to our clinic for global development delay and lack of speech. He was hypotonic and has been avoiding eye contact since the age of six months. He had a history of generalized epileptic seizures from the age of 6 months, which responded well to valproic acid. One year post medication, he began to have secondary generalized epileptic seizures, which ceased after addition of levetiracetam to valproic acid. However, seizures recurred 20 months later. The patient had a family history of epilepsy with intellectual disabilities present in his elder sister and his aunt.

On physical examination, the patient had delayed neuromotor development and intellectual disabilities. Dysmorphic features included narrow and upslanted palpable fissures, thin sparse hair, long smooth philtrum, posterior cleft palate, broad mouth with tented thin upper lip, widely spaced nipples, and brachytelephalangy. He had generalized hypotonia. He also had autistic features with no eye contact.

Laboratory tests revealed normal complete blood count (CBC), normal serum biochemistry including calcium, phosphorus, and magnesium. Vitamin D levels, metabolic screening and thyroid functions were normal. However, there was markedly elevated serum alkaline phosphatase levels at $905 \mathrm{IU} / \mathrm{L}$ and $771 \mathrm{IU} / \mathrm{L}$ at the age of three and four and, was still elevated to the level of $566 \mathrm{IU} / \mathrm{L}$ (normal range: 40-150 IU/L) at the age of six years. Moreover, electroencephalogram (EEG) test revealed active generalized epileptic seizures along with severe bioelectric cerebral dysfunction which was in consistence with the previous two EEGs at the age of three years and four years.

\section{Case 2}


The elder sibling was a 14-year-old girl who was referred to our clinic for investigation of epileptic seizures. At five years of age, she was noted to have global development delay. The patient also developed seizures at the age of five. Carbamazepine was prescribed but it was arbitrarily stopped by the parents. The frequency of seizures, which previously was at 1-2 times a year, gradually increased to 1-2 times a month that lasted for 3-5 minutes each. Valproic acid was administered. However, the seizures recurred after 12 months. Valproic acid was then stopped and levetiracetam was administered. Then lacosamide was used together with levetiracetam. However, this did not suppress the seizures and the frequency of seizures increased to 3-5 times a month that lasted for 5-10 minutes each.

On physical examination, the patient had delayed neuromotor development and intellectual disabilities. She had generalized hypotonia and is not able to walk until now. Deep tendon reflexes were significantly increased in all four extremities. Dysmorphic features included long and upslanting palpebral fissures, broad nasal bridge with squared nasal tip, a tented upper lip with downturned corners of the mouth, a high-arched palate and brachytelephalangy.

Laboratory tests revealed normal complete blood count (CBC), normal serum biochemistry including calcium, phosphorus, and magnesium. Vitamin D levels, parathormone hormone and thyroid functions tests were normal. Metabolic screening was normal. However, Serum alkaline phosphatase (ALP) level was elevated with 682 IU/L at fourteen years age and, was still elevated with $496 \mathrm{IU} / \mathrm{L}$ (normal range: 40-150 IU/L) at sixteen years of age. In addition, electroencephalography (EEG) examinations showed multi-focal onset epileptic seizures.

\section{Mutation Testing}

The family history of dysmorphic features along with persistent epileptic seizures, led to further biochemical investigations, and subsequently to whole exome sequencing genetic testing. Consequently, a homozygous novel missense variant in a well-conserved region, c.1003G $>$ A (p. Ala335 Thr chr11.3,846,572 NM_001256236.1), was identified in the exon 8 of PGAP2 gene by whole-exome sequencing in both of our patients.

\section{Discussion}

We report 2 Turkish patients with Mabry syndrome, which was diagnosed clinically and genetically, both with homozygous PGAP2 mutations.

There are mutations in six genes ( $P I G V$, PIGY, PIGO, PGAP2, PIGW, and PGAP3) that have been responsible for hyperphosphatasia with mental retardation syndrome (HPMRS) reported until now. All of these genes are involved in GPI-anchor biosynthesis [1][2]. While PIGV and PIGO are the two most common genes reported related to Mabry syndrome, PGAP2 mutations are rare [6]. PGAP2 is a non-catalytic protein which assists in attaching a saturated fatty acid to the anchor. This function of PGAP2 occurs in the Golgi apparatus and is required for stable association between GPI-anchored proteins and the cell-surface membrane rafts [4]. GPI-anchored proteins play an important role in membrane protein trafficking, cell-surface adhesion, and protection [3]. ALP, a GPI anchor protein, is important for neuronal development and plays a major role in vitamin B6 (pyridoxine) metabolism [5].

PGAP2 gene mutation is responsible for HPMRS3, which presents with varying degrees of mental retardation and elevated ALP. Less commonly reported symptoms include epilepsy, hypotonia, microcephaly and dysmorphism [1][2]. The consistent dysmorphic features include narrow palpable fissures, broad nasal bridge, large fleshy ear lobes, long smooth philtrum, tented upper lip, cleft palate, along with behavioral problems and lack of speech, which were in consistence with our patients [4]. It is worth noting that both of our patients had axial hypotonia, increased deep tendon reflexes and spasticity, which indicate spastic quadriplegia is a novel feature in both of our patients. Inverted nipples were the other novel dysmorphic feature seen in both of our patients.

Our reported patients showed homozygous novel missense variant in a well conserved region c.1003G $>$ A (p. Ala335 Thr chr11.3,846,572 NM_001256236.1) which has not been reported before in mutation database.

The GPI-APs expression levels are associated with the location of mutation on the chromosome; and also, the severity of the phenotypic features correlates with the level of conservation of the mutation [1][3]. However, further PGAP2 mutational reports in different patients are required to confirm this observation. In the current study, we report the 
clinical and molecular findings of two new patients with HPMRS with PGAP2 mutations. The patient presented with the classical phenotypic and distinct facial features of the disorder. These facies together with the generalized epileptic seizures and elevated serum alkaline phosphatase are significant indicators for targeting genetic testing of PGAP2.

In conclusion, global developmental delay and facial dysmorphism in the presence of elevated alkaline phosphatase is a strong predictor of HPMRS. This case report also highlights the successful application of exome sequencing in consolidating the diagnosis which is consistent with previously reported cases. The novel mutations of PGAP2, and the clinical manifestations reported here, are significant in expanding the genotype and phenotype spectrum of Mabry syndrome.

\section{Funding}

No funding was received to assist with the preparation of this manuscript.

\section{Conflict of Interest}

The authors have no conflicts of interest to declare that are relevant to the content of this article.

\section{Informed consent}

Informed consent was obtained from the patient and parents.

\section{References}

1. Wu T, Yin F, Guang S, He F, Yang L, Peng J. The Glycosylphosphatidylinositol biosynthesis pathway in human diseases. Orphanet J Rare Dis. 2020 May 28;15(1):129. doi: 10.1186/s13023-020-01401-z. PMID: 32466763; PMCID: PMC7254680.

2. Perez Y, Wormser O, Sadaka Y, Birk R, Narkis G, Birk OS. A Rare Variant in PGAP2 Causes Autosomal Recessive Hyperphosphatasia with Mental Retardation Syndrome, with a Mild Phenotype in Heterozygous Carriers. Biomed Res Int. 2017;2017:3470234. doi:10.1155/2017/3470234

3. Murakami Y, Kinoshita T. [Inherited GPI deficiencies:a new disease with intellectual disability and epilepsy]. No To Hattatsu. 2015 Jan;47(1):5-13. Japanese. PMID: 25803904.

4. Knaus, Alexej et al. "Rare Noncoding Mutations Extend the Mutational Spectrum in the PGAP3 Subtype of Hyperphosphatasia with Mental Retardation Syndrome." Human mutation vol. 37,8 (2016): 737-44. doi:10.1002/humu.23006

5. Kuki I, Takahashi Y, Okazaki S, Kawawaki H, Ehara E, Inoue N, Kinoshita T, Murakami Y. Vitamin B6responsive epilepsy due to inherited GPI deficiency. Neurology. 2013 Oct 15;81(16):1467-9. doi: 10.1212/WNL.0b013e3182a8411a. Epub 2013 Sep 18. Erratum in: Neurology. 2013 Nov 12;81(20):1803. PMID: 24049131.

6. Xue, J., Li, H., Zhang, Y., \& Yang, Z. (2016). Clinical and genetic analysis of two Chinese infants with Mabry syndrome. Brain and Development, 38(9), 807-818. doi:10.1016/j.braindev.2016.04.008 